\title{
LAS MULTINACIONALES, AGENTES ESTRATÉGICOS DEL CAPITAL. UNA GUÍA PARA EVALUAR SUS IMPACTOS
}

\author{
MULTINATIONALS, STRATEGIC AGENTS OF CAPITAL. A GUIDE FOR \\ ASSESSING THEIR IMPACT
}

\author{
Luis Miguel Uharte \\ Universidad del País Vasco (UPV/EHU). España/Spain \\ luismiguel.uharte@ehu.es
}

Recibido/Received: 27/03/2014

Modificado/Modified: 30/05/2014

Aceptado/Accepted: 27/06/2014

\section{RESUMEN}

El objeto de este trabajo ha sido construir una guía de análisis que sirva a los investigadores para poder evaluar los impactos múltiples que las corporaciones multinacionales provocan habitualmente con su práctica empresarial. En primer lugar, se plantea de manera breve una definición de este tipo de empresas y la evolución que han tenido a lo largo de la historia. A continuación se señalan sus atributos más importantes y su actual distribución a escala global. Finalmente, el capítulo central del texto se ocupa de identificar los impactos más importantes que las multinacionales generan en diversos ámbitos, destacándose entre otros la mercantilización de servicios públicos, la pérdida de soberanía, la práctica del lobby y la corrupción, la criminalización social, el deterioro medioambiental y la vulneración de derechos laborales. En síntesis, estas grandes corporaciones privilegian su función como agentes estratégicos del capital por encima de otros fines sociales.

\section{PALABRAS CLAVE}

Mercantilización de servicios públicos, soberanía nacional, corrupción, deterioro ambiental, vulneración de derechos, pueblos indígenas.

\section{SUMARIO}

1. Introducción. 2. Definición y evolución histórica. 3. Atributos y distribución. 4. Impactos. 5. Conclusiones. Bibliografía.

\begin{abstract}
The main objective of this work has been to build an analysis guide that serve researchers to evaluate the multiple impacts that multinational corporations usually cause your business practice. First, it presents briefly a definition of these businesses and developments that have had throughout history. Then their most important attributes and their current global distribution are indicated. Finally, the central chapter of the text is concerned with identifying the major impacts that multinationals generate in various fields, highlighting among others the commodification of public services, loss of sovereignty, the practice of lobbying and corruption, social criminalization, deterioration environmental and labor rights violations. In summary, these large corporations favor its role as strategic agents of capital over other social purposes.
\end{abstract}




\section{KEYWORDS}

Commodification of public services, national sovereignty, corruption, environmental damage, violation of rights, indigenous peoples

\section{CONTENTS}

1. Introduction. 2. Definition and historical evolution. 3. Features and distribution. 4. Impacts. 5. Conclusions. References.

\section{INTRODUCCIÓN}

La literatura sobre el fenómeno de las multinacionales es extensa y dilatada en el tiempo. Los análisis sobre su práctica empresarial son numerosos, tanto los realizados por grupos de investigación vinculados a este tipo de empresas (fundaciones adscritas o financiadas por estas) como por los de investigadores que se sitúan en posiciones críticas (universidad, movimientos sociales...). Sin embargo, se puede afirmar que los posicionamientos favorables a estas grandes corporaciones suelen ser mayoritarios por la evidente dependencia que generan a través del control directo (propiedad) o indirecto (financiación) de centros de investigación y fundamentalmente de los grandes medios de comunicación.

De cualquier manera, las investigaciones críticas sobre las multinacionales no son escasas, a pesar de las limitaciones que en muchos casos suelen padecer, principalmente de carácter financiero y de recursos humanos. Se han realizado trabajos relevantes sobre los efectos que provocan en diversos ámbitos, no solo en el estrictamente económico, e incluso algunos autores han elaborado guías para evaluar algunos impactos que provocan estas empresas, pero consideramos que es sumamente complicado encontrar una guía que mida impactos múltiples, es decir, de orden económico, político, social, ambiental y cultural. En consecuencia, el objetivo fundamental de este trabajo es diseñar una herramienta de trabajo para los investigadores que estén interesados en evaluar de manera integral los diversos impactos que las multinacionales generan con su práctica empresarial. Somos conscientes que no aparecen todos los efectos que provocan pero consideramos que en general el grupo de dimensiones identificado permite un análisis múltiple.

El texto comienza con un apartado dedicado a definir las corporaciones multinacionales y a presentar brevemente su evolución y transformaciones a lo largo de la historia. Posteriormente se señalan las principales características de estas empresas y los países más importantes en cuanto a su distribución y origen. A continuación, el apartado más extenso se ocupa de identificar los impactos más importantes que las multinacionales generan en diversos ámbitos, destacándose entre otros la mercantilización de servicios públicos, la pérdida de soberanía, la práctica del lobby y la corrupción, la criminalización social, el deterioro medioambiental y la vulneración de derechos laborales. Estos impactos identificados componen la guía de análisis que proponemos para evaluar la práctica de las corporaciones transnacionales, que aparece resumida en la tabla que elaboramos al final del texto. Finalmente, reservamos un último apartado a presentar una serie de conclusiones.

\section{DEFINICIÓN Y EVOLUCIÓN HISTÓRICA}

Leslie Sklair (2003:69) asegura que el término corporación multinacional fue acuñado en 1960 por David Lilienthal, alto funcionario del gobierno de los Estados Unidos, con la 
publicación del texto The Multinational Corporation, a pesar de que la existencia de este tipo de organización empresarial se hiciera sentir mucho antes en la historia. Lilienthal planteaba en ese momento una definición marcadamente aséptica y sencilla: "empresas que tienen su sede en un país pero que operan y funcionan de acuerdo con leyes de otros países". Agregaba que las multinacionales cuentan con una base productiva o alguna forma de inversión directa en un país extranjero y que su perspectiva es auténticamente mundial a la hora de tomar decisiones estratégicas.

Sin embargo, ya desde la década de los setenta, las definiciones y los enfoques sobre el papel que las multinacionales desempeñaban en el mundo contemporáneo eran notablemente antagónicos. Un informe publicado por Business Week en estos años indicaba que estas corporaciones promovían el crecimiento, desarrollaban recursos inutilizados, daban nuevas oportunidades al talento y ayudaban a "mejorar el nivel de vida en las naciones industrializadas y en los países en desarrollo". Por el contrario, investigadores como Stephen Hymer aseguraban que las multinacionales eran agentes de dominación y explotación, de generación de dependencia y herramientas del imperialismo (Teitelbaum, 2010:17-8).

Unas décadas después, en el actual contexto de hegemonía del capitalismo global de principios del siglo XXI, las definiciones sobre estas sociedades siguen siendo antagónicas en función de la procedencia del análisis. Desde los ámbitos de investigación vinculados a las grandes empresas se les sigue considerando agentes centrales para el desarrollo económico mundial (Guillen, 2006; Casilda, 2008), mientras que los investigadores más críticos caracterizan a las multinacionales como las "unidades operativas básicas del mundo capitalista y del imperialismo" (Petras y Veltmeyer, 2007:63).

Lo que parece cada vez más evidente es su papel central en la dinámica del funcionamiento del actual sistema, como apuntan diversos expertos: Achbar y Abbot (2009) consideran que son "la institución dominante de nuestro tiempo"; Carrión y Verger (2007:10) afirman que "son el actor económico más relevante del sistema global actual", ya que están detrás del $85 \%$ de la Inversión Extranjera Directa (IED) y del 66\% del comercio mundial; el catedrático de la Universidad de Barcelona Joan Sánchez Pérez (2008), por su parte, asevera que representan "uno de los bloques de poder más importantes a escala mundial, en la medida en que su volumen de actividad, así como su capacidad de incidir sobre las economías nacionales, su extensión sobre el conjunto del territorio mundial, y la concentración en relativamente pocas manos de su poder de decisión, hacen de ellas uno de los ámbitos de intervención más potentes que podemos encontrar en este momento".

Para poder dimensionar y sobre todo entender el poder actual de las multinacionales es necesario comprender el marco en el que están insertas, es decir, el sistema capitalista, y la evolución histórica de éste desde el surgimiento de las primeras corporaciones de carácter transnacional. Algunos autores ubican su nacimiento en el contexto del colonialismo de los siglos XVII y XVIII, refiriéndose a las compañías comerciales que expoliaban e importaban "grandes cantidades de metales preciosos de las colonias" (Carrión y Verger, 2007:10). Otros sitúan el origen en la segunda mitad del siglo XIX, en el marco de la primera fase imperialista del capitalismo moderno, con su proceso de concentración de propiedad y apropiación de recursos de la periferia. Un ejemplo paradigmático sería la Standard Oil, fundada por Rockefeller en 1870 (Teitelbaum, 2010:36).

El cambio de modelo que ocurre al interior del capitalismo tras la "crisis del 29", con la sustitución del liberalismo por una propuesta más estatista de corte keynesiano, incide directamente en la composición de las multinacionales. De hecho, será en los años cuarenta, tras el fin de la segunda guerra mundial, cuando surja "la moderna empresa transnacional", 
donde el modelo predominante será "la gran empresa estadounidense con filiales en Europa y en Latinoamérica", que fabrica productos similares a los de la casa matriz, destinados al mercado local (Zabalo, 2007:7). En ese contexto de capitalismo keynesiano, la actividad industrial y comercial de las grandes empresas será el "rasgo dominante" de su funcionamiento (Teitelbaum, 2010:36).

La reorganización capitalista de la década de los setenta, con la implantación progresiva del neoliberalismo, creará el terreno ideal para el crecimiento y el fortalecimiento de las multinacionales. Las privatizaciones de recursos públicos estratégicos tanto en los países del Norte como del Sur, el retroceso del Estado en el campo económico, la concentración constante de propiedad empresarial, la hegemonía del capital financiero y los diversos cambios normativos, son algunos de los factores que permitieron aumentar exponencialmente el poder de las transnacionales. Si en los años setenta había unas 7.000 grandes empresas, tres décadas después se multiplicaron por diez, superando la cifra de 70.000 , y con filiales que llegan a alcanzar la cifra de alrededor de 700.000 en todo el mundo (Zabalo, 2007:7).

Es importante señalar que la denominada globalización adoptó un "diseño neoliberal" para garantizar el "beneficio privado" a través de la creciente "mercantilización de nuevos sectores y actividades". Mediante la privatización de servicios básicos para la comunidad se "abren nuevos campos para el negocio" de las empresas transnacionales: electricidad, telecomunicaciones, agua, etc. Paralelamente, las reformas legales en función de los intereses de las grandes corporaciones posibilitan que estas absorban a pequeñas y medianas empresas de diversos sectores productivos en todo el mundo. Se produce así otra de las consecuencias "esenciales de la globalización", la concentración "del poder económico en cada vez menos manos" (Hernández Zubizarreta, 2009b:111-3).

\section{ATRIBUTOS Y DISTRIBUCIÓN}

Diversos rasgos de carácter económico atestiguan el poder actual de las multinacionales, a inicios del siglo XXI, y su condición de "institución dominante de nuestro tiempo". El conjunto de las 500 mayores transnacionales acumulan un volumen de negocio y de número de empleados sumamente relevante. Con relación al conjunto del PIB mundial, según los datos del FMI, los ingresos de las 500 mayores han pasado de representar el equivalente al $38 \%$ en 1996 al $43 \%$ en 2006, es decir, casi la mitad de la riqueza mundial. Superan por tanto el PIB de EE.UU. o el de la Unión Europea. En cuanto al número de personas que emplean, según los datos de 2006 tenían a su servicio a casi 53 millones de trabajadores, lo que supone un número mayor de empleados que países como Alemania, Reino Unido o Francia (Sánchez Pérez, 2008).

El poder económico de las multinacionales es aún mucho más evidente si lo comparamos con la mayoría de los países del Sur: en 2004, por ejemplo, "General Motors facturó 191.400 millones de dólares, cifra mayor que el PIB de más de 148 países"; en 2005, los grandes almacenes "Wal-Mart tuvieron unos ingresos de 285.200 millones de dólares, es decir, más que el PIB combinado de todos los países de África Subsahariana” (Hernández Zubizarreta, 2009b:113).

Este poder de riqueza concentrado en "500 centros de decisión" deja claro no solo el "peso directo sobre el sistema productivo mundial", sino también su "capacidad de influencia directa y derivada sobre el resto del sistema". Su incidencia en el poder político es manifiesta ya que constituyen auténticos "poderes fácticos que presionan para que los instrumentos del 
Estado sirvan a sus intereses estratégicos", tanto en el país-sede como en los territorios donde tienen filiales (Sánchez Pérez, 2008).

Algunas lecturas posmodernas tratan de caracterizar a las actuales multinacionales como entes abstractos y desterritorializados, donde sería imposible identificar a sus responsables. Sin embargo, a pesar de que el movimiento de acciones, fusiones y absorciones es constante, lo que sí se mantiene más estable son "los nombres de las familias que, como nuevas dinastías, son las mayores accionistas de las grandes corporaciones económicas. 225 familias de entre los multimillonarios del planeta poseen fortunas superiores a los ingresos anuales de 2.500 millones de personas. Sus desproporcionados ingresos les permiten no sólo concentrar parte del capital mundial, sino ejercer un importantísimo poder político y económico" (Van den Eynde, Cohen y Ligammari, en Hernández Zubizarreta, 2009b:118).

En cuanto a la distribución internacional de las grandes corporaciones (las 500 más grandes de la lista Fortune), la "triada" (EE.UU., Europa y Japón) sigue concentrando la mayoría de empresas y de beneficios, a pesar de que va retrocediendo de manera constante. Japón es quien retrocede de forma más acelerada, pasando de 126 en 1996 (un 25\% del total mundial) a 67 en 2006 (un 13\%). Para el año 2006, Estados Unidos y Europa retenían el $32,4 \%$ (162) y el 34,2\% (178) del total de empresas, respectivamente, lo que evidencia que los países del capitalismo central siguen teniendo el control mayoritario. A esto hay que añadir que en ese mismo año, entre los dos sumaban el $76 \%$ de los beneficios del total de las 500, lo cual corrobora aún más su hegemonía (Sánchez Pérez, 2008).

De todas formas, esto no oculta el incremento exponencial de las multinacionales de China, India y Sud-Asia (Santiso, 2009). En el caso chino, de 1996 a 2006 el aumento del número de corporaciones que ingresaron en el club de las 500 más grandes fue del $380 \%$, pasando de 5 a 24. El aumento de sus beneficios y de empleados fue astronómico, por encima de $3.000 \%$ y de $1.500 \%$, respectivamente (Sánchez Pérez, 2008).

Para el año 2011, esta tendencia se acentúa, ya que China ocupa el tercer lugar mundial, con 61 corporaciones, sólo por detrás de Japón (68) y de Estados Unidos (133). Brasil, otro de los miembros del BRIC, dispone ya de 7 transnacionales en la lista, cerca de las 9 que representan al Estado español: Santander, Telefónica, Repsol, BBVA, Iberdrola, Cepsa, Gas Natural-Fenosa, Mapfre y ACS (Basterra, 2011).

Otra tendencia destacable de los últimos años es el aumento sustancial, dentro de la citada lista de las 500 más grandes, de las corporaciones mineras y petroleras -que pasan de 34 a 49 y de las productoras y distribuidoras de energía y agua -que pasan de 17 a 30-. El crecimiento está íntimamente relacionado, según Sánchez Pérez (2008), con la dinámica "especulativa a la que han estado sometidas estos años" las materias primas. A esto deberíamos agregar otro factor, principalmente en lo que concierne a los servicios de energía y agua, y es el negocio fabuloso que se abre con la privatización y la consecuente concentración de propiedad que se traduce en la constitución de grandes corporaciones.

\section{IMPACTOS}

La actividad de las multinacionales genera una serie de impactos múltiples, no solo en el campo económico, sino también en otros ámbitos, como el político, el social, el laboral, el ambiental, el cultural, etc. En relación a este asunto, las lecturas son de nuevo notablemente divergentes según la procedencia del análisis. Desde los grupos de investigación cercanos y/o vinculados a las corporaciones se subrayan los impactos positivos que estas generan en 
términos de "desarrollo" y que se traduce en: inversión económica, impulso a la actividad empresarial, generación de empleo, buenas condiciones laborales, mejora de la calidad de los servicios, reducción de precios de productos de consumo masivo y de los servicios públicos, etc. (Casilda, 2008a; Guillén, 2006).

Desde los espacios de investigación críticos, se pone el acento en los impactos negativos que provoca la actividad de las multinacionales, tanto en los países del Norte como en los del Sur. Se considera además, que existe un desequilibrio en el campo de la investigación sobre la práctica de las grandes corporaciones, ya que estas destinan importantes recursos para investigaciones que avalen su actividad, mientras que las investigaciones críticas disponen de un volumen de financiación mucho más limitado. En consecuencia, termina siendo muy complicado contrarrestar la publicidad, el marketing y los análisis complacientes en torno a las transnacionales.

A continuación vamos a presentar diversos impactos que provocan las corporaciones transnacionales en los lugares donde operan. Los impactos identificados pretenden configurar una guía de elaboración propia, que sea válida para abordar análisis y evaluaciones sobre las prácticas de las empresas multinacionales.

\section{1. Área económica}

1. Mercantilización. Los diversos impactos económicos que generan las corporaciones en su actividad empresarial constituyen una de las dimensiones más importantes a la hora de evaluar su funcionamiento. Uno de los impactos de mayor relevancia está relacionado con el modelo de propiedad que defienden las compañías y las consecuencias que esto tiene para la sociedad en su conjunto. El caso más paradigmático es el de la propiedad de los recursos estratégicos y de los servicios públicos, es decir, del petróleo, el gas, los minerales, la electricidad, el agua, las telecomunicaciones... Desde la década de los noventa, la privatización y en muchos casos la apropiación por parte de multinacionales de recursos y servicios básicos como la generación y la distribución de energía eléctrica, el servicio de gas, las telecomunicaciones, la provisión de agua, etc., han provocado la conversión de un "bien público" en una "mercancía". Esto implica que la "lógica de lucro" se torna prioritaria frente a la "lógica de asegurar un servicio público básico a toda la población", independientemente de su condición social. Esto supone que el privilegio de explotar un nuevo negocio puede llegar a estar por encima de garantizar derechos humanos y sociales básicos, como la provisión de luz eléctrica y agua (Uharte, 2005:109-110).

2. Soberanía nacional. Otro de los impactos de mayor relevancia en el terreno de la economía es el relativo a la pérdida de soberanía nacional. La venta de un recurso público a corporaciones foráneas no supone solo la privatización de un sector estratégico sino también la desnacionalización o la extranjerización de este. Se "lesiona severamente la soberanía nacional" cuando las "industrias estratégicas" se convierten en propiedad extranjera, privando al Estado de la potestad de tomar decisiones en áreas clave (Petras y Veltmeyer, 2007:223).

El contra-argumento que utilizan los defensores de la privatización desnacionalizadora es que se produce un efecto positivo porque se estimula la competencia en el sector. Sin embargo, en muchos casos lo que ocurre es una sustitución de un régimen de monopolio público por otro privado y en otros una situación de oligopolio, que de facto suprime la potencial competencia. En este campo, son diversos los ejemplos donde las pequeñas empresas locales terminan cerrando debido a la competencia desigual que tienen que padecer frente a la poderosa corporación extranjera (Van den Eynde, 2005). 
Otro argumento habitual a favor de la llegada de las multinacionales es la supuesta creación de nuevo tejido industrial y productivo y a su vez de fomentar la transferencia tecnológica. En la práctica, por el contrario, y en el caso de América Latina de forma más acusada, generalmente compran empresas ya existentes en sectores rentables que estaban bajo control del Estado, como los antes mencionados: electricidad, telecomunicaciones, etc. (Petras y Veltmeyer, 2007:225).

3. Rentabilidad. Los elevados márgenes de rentabilidad que obtienen muchas empresas transnacionales se convierten en otro aspecto relevante de los impactos económicos, ya que estas elevadas ganancias se producen en detrimento del erario público y/o de gran parte de la población. Hay diversos mecanismos para que se produzca esta trasferencia de riqueza de manos públicas a privadas, a veces operando de manera aislada y en otros casos de forma combinada. Algunos de estos mecanismos serían los siguientes: privatización de recursos públicos a bajo costo, legislación que permite altos precios del servicio, subvenciones públicas y ventajas fiscales (reducción de impuestos), entrada en negocios regulados donde el Estado asegura la compra, imposición de salarios bajos, explotación intensiva de la fuerza de trabajo, etc. (Petras y Veltmeyer, 2007:228).

4. Eficiencia. El nivel de efíciencia es una de las aristas de la dimensión económica que mayor controversia genera, ya que el discurso neoliberal de los años noventa aseguraba que la privatización de los servicios públicos traería como consecuencia la mayor eficiencia en la gestión de las antiguas empresas estatales. Aunque esto pudiera ser cierto en algunos casos, en otros ocurre todo lo contrario, ya que la privatización provoca la fragmentación de un servicio con la aparición de diferentes empresas y la consecuente duplicación de infraestructuras, como ocurre en el ramo de la electricidad (Barba, 2008:8-9). Otro exponente de la ineficiencia del servicio privatizado sucede cuando la multinacional no invierte lo suficiente en infraestructuras y en el mantenimiento de las redes, provocando un deterioro del sistema y un aumento de los cortes, apagones, etc. (Ramiro, 2011).

En el área económica, un último aspecto que quisiéramos destacar es la relación que se establece entre las multinacionales y sus proveedores, ya que en algunos casos se da prioridad a empresas externas y no a las locales, impactando negativamente en el tejido empresarial autóctono. A su vez, otro impacto negativo se produce cuando la gran corporación contrata con proveedores que no respetan los derechos laborales y humanos, sustentando y siendo cómplice de situaciones de explotación y/o represión.

\section{2. Área política}

1. Democracia. La actividad de las transnacionales no solo provoca impactos en el terreno económico sino también en otros campos como el político. Uno de ellos tiene que ver directamente con su posicionamiento ante la democracia, ya que en más de una ocasión han estado detrás de golpes de Estado y/o beneficiándose de regímenes dictatoriales como acertadamente nos recuerda Teitelbaum (2010: 81-4): "son incontables los ejemplos que se podrían dar en materia de participación de sociedades transnacionales en golpes de Estado y sobre su estrecha colaboración con dictaduras". Entre otros, destacan el apoyo de las compañías petroleras al golpe en Irán en 1953, el papel de la United Fruit Company en el golpe contra Arbenz en Guatemala en 1954, la participación de la International Telephone and Telegraph (ITT) en el derrocamiento de Salvador Allende en Chile en 1973, el apoyo a la dictadura argentina (1976-1983) de corporaciones como Fiat, Renault, Ford... En esta misma línea, Howard Zinn y Noam Chomsky coinciden en vincular el ascenso del nazismo en Europa con el apoyo de las grandes corporaciones (en Achbar y Abbot, 2009). 
Existen otras vías menos agresivas y explícitas que las multinacionales han utilizado y utilizan para afectar al funcionamiento del sistema democrático. Una de ellas es incidir "en los procesos electorales de los países en los que se encuentran ubicadas", inyectando por ejemplo, "grandes sumas de dinero en las campañas electorales de los partidos políticos más afines" o "transmitiendo una versión negativa a la opinión pública de lo que sucederá en el país si sube al poder el candidato que menos les interesa" (Carrión y Verger, 2007:12).

2. Lobby. La práctica habitual del lobby por parte de las multinacionales es uno de los impactos políticos de mayor trascendencia, que obviamente deteriora la democracia. Hernández Zubizarreta (2009b:132) advierte de los "múltiples recursos económicos" que las corporaciones invierten para influir en las decisiones políticas tanto de organismos nacionales como internacionales. El lobby empresarial europeo es un buen ejemplo de ello, con más de mil grupos de presión, cientos de empresas de relaciones públicas, despachos de abogados y con una facturación anual cercana a los 1.000 millones de euros. Balanya (2007:23) alerta de los recurrentes casos de antiguas autoridades políticas y ex altos funcionarios que pasan a formar parte posteriormente del lobby de las grandes firmas, como el caso del ex Comisario de Comercio de la Unión Europea, Leon Brittan, que pasó a formar parte del grupo de presión que representa a la industria británica de servicios financieros. Teitelbaum (2010: 155-9), por su parte, apunta al lobby transnacional que se incrusta en la ONU desde la década de los noventa, principalmente con la creación del "Global Compact" donde participan 44 grandes corporaciones para promover la privatización y la desregulación generalizada a escala internacional.

3. Mass media. Un impacto relevante en términos políticos y democráticos es la incidencia que las transnacionales tienen en los grandes medios de comunicación. Carrión y Verger (2007: 13) apuntan que hoy día, los mass media "no acostumbran a informar" sobre los "abusos que se derivan de la actividad" de las multinacionales, porque tienen "una estrecha relación con ellas": por una parte, por la dependencia de algunos medios de la publicidad que estas insertan y por otra, porque las corporaciones directamente tienen acciones en los medios. De hecho, como recuerda Teitelbaum (2010:117), actualmente estamos en la época de la concentración oligopólica de los medios de comunicación, donde grandes corporaciones tienen el control mundial de la información y el entretenimiento, como General Electric (NBC- Vivendi Universal), AOL-Time Warner, AT\&T Corp, Viacom Inc., Walt Disney, News Corp, Bertelsmann, Sony y Liberty Media Corp". Los dos mayores fabricantes de armas de Francia (Dassault y Lagardère), por ejemplo, "controlan casi toda la prensa francesa". La consecuencia de todo esto, como acertadamente señala Sklair (2003: 304), es que las visiones críticas van a tener una capacidad mucho más limitada para contrarrestar el apoyo de las grandes corporaciones mediáticas a las transnacionales, evidenciando una situación de absoluto desequilibrio, como apuntábamos anteriormente.

4. Derecho comercial global. Uno de los impactos de mayor trascendencia en el ámbito político tiene que ver con el nuevo marco jurídico favorable a las multinacionales, que se ha ido configurando a nivel internacional producto de la presión permanente de estas, como señalan diferentes autores (Hernández Zubizarreta, 2008 y 2009b; Teitelbaum, 2010; Giner, 2008; Zabalo, 2007). Hernández Zubizarreta (2009a:20-3) afirma que "es flagrante la asimetría entre la evolución del derecho comercial global y la del derecho internacional de los derechos humanos". De un sistema normativo y obligatorio se ha ido pasando a otro "voluntario y unilateral". Un ejemplo paradigmático de este cambio se ha producido en la Organización Internacional del Trabajo (OIT), donde se han ido sustituyendo las normas obligatorias (los convenios) por las recomendaciones. 
La filosofía de la voluntariedad ha tenido su traducción más expresa en la creación de la llamada "Responsabilidad Social Corporativa", que ha pasado "a convertirse en el nuevo paradigma del comportamiento de las empresas multinacionales en la era de la globalización" (Ramiro, 2009:48). Las empresas diseñan sus códigos de conducta totalmente voluntarios para ganar "legitimación social", sabiendo que una infracción no va a tener consecuencias legales (Ramiro, 2009:73). Esto supone la implantación de la filosofía del Soft Law, según la cual las buenas prácticas empresariales "dependen totalmente de la buena voluntad de las propias empresas" (Giner, 2008:76).

Paralelamente a la lógica de la voluntariedad se han realizado transformaciones legales para proteger a las grandes corporaciones frente a las propias instituciones públicas. La creación de tribunales al margen del sistema judicial ordinario es un dato relevante en este sentido, como ha ocurrido con la constitución del CIADI (Centro Internacional de Arreglo de las Diferencias en Inversiones). Este es un órgano que impulsó el Banco Mundial para dirimir los conflictos sobre inversiones entre multinacionales y Estados. Teniendo en cuenta que el $80 \%$ de sus fondos provienen de las corporaciones, no resulta extraño que se le haya tachado de imparcial cuando en la mayoría de los casos ha fallado en contra de las instituciones públicas (Teitelbaum, 2010:203).

5. Corrupción. La corrupción es otro de los impactos políticos ligado a las prácticas de las multinacionales, fundamentalmente a través del soborno a agentes públicos, funcionarios, altos cargos gubernamentales, etc., a los que se les promete una remuneración a cambio de legislar a su favor, adjudicar contratos de obra o servicio, conseguir un trato de privilegio... (Carrión y Verger, 2007:12). Al ser una práctica "oculta" resulta difícil de medir, aunque la OCDE (Organización para la Cooperación y el Desarrollo Económico) calcula que el soborno internacional se acerca los 150.000 millones de dólares al año en todo el mundo, mientras que Transparencia Internacional lo eleva a los 400.000 millones. Multinacionales sobradamente conocidas como la petrolera Total tienen "varios sumarios abiertos por la justicia francesa relacionados con comisiones ocultas y blanqueos de dinero" y "corrupción de funcionarios públicos". Las privatizaciones en América Latina en los años noventa "estuvieron rodeadas de numerosos casos de corrupción". Por ejemplo, en Nicaragua el gobierno de Violeta Chamorro vendió "341 de las 351 empresas estatales por debajo del 75\% de su precio de mercado" (Hernández Zubizarreta, 2009b:128-9), un buen número de ellas a transnacionales.

6. Transparencia. Otro impacto de orden político que quisiéramos subrayar sería la falta de transparencia con la que actúan las grandes corporaciones en determinados casos. La dificultad para obtener información clara de sus movimientos y ayudas públicas, la presencia recurrente en paraísos fiscales y el uso de la publicidad y el marketing para proyectar una imagen distorsionada de su funcionamiento (Teitelbaum, 2010:241), son algunas de las variables que certifican esta falta de transparencia.

7. Criminalización social. La criminalización y represión de grupos de trabajadores, movimientos sociales, etc. que se oponen a las prácticas de las multinacionales supone un impacto tanto en el apartado de los derechos humanos como en términos de derechos políticos, y se convierte en un indicador importante a la hora de evaluar el funcionamiento de las corporaciones. Ramiro (2011:11) recuerda que "en muchos casos, los integrantes de las organizaciones sociales que han protagonizado movilizaciones populares y que han criticado la presencia de corporaciones transnacionales en sus territorios han sido criminalizados y, en ocasiones, han sido duramente reprimidos por la fuerza pública o por milicias paramilitares. $\mathrm{Y}$ es que, desgraciadamente, resulta muy común que las áreas donde tienen intereses las 
multinacionales -por ejemplo, aquellas en las que se llevan a cabo explotaciones mineras o hidrocarburíferas- estén militarizadas y se produzcan atentados contra los activistas sociales y los defensores de los derechos humanos".

\section{3. Área ambiental}

Los diversos impactos ambientales constituyen una dimensión de gran relevancia en la práctica diaria de muchas transnacionales. En primera instancia, resulta necesario plantear una lectura histórica del papel que las grandes corporaciones han jugado a lo largo del capitalismo como agentes centrales en la generación de la deuda ecológica en los países del Sur. Como bien sabemos, la "deuda ecológica es la deuda contraída por los países industrializados con los demás países a causa del expolio histórico y presente de los recursos naturales, los impactos ambientales exportados y la libre utilización del espacio ambiental global para depositar los residuos". Reflejaría lo que el Norte debe al Sur por esta degradación producida y que hoy continúa (Bárcena y Lago, 2008:147).

1. Contaminación. Las diversas y múltiples formas de contaminación constituyen uno de los tipos de impacto ambiental más evidentes y significativos. La contaminación del aire, del agua, de la tierra, de la flora, de la fauna y de los seres humanos, es una consecuencia directa del modelo desarrollista en el que estamos insertos y que se padece tanto en los países del Norte como en los del Sur y en el que las grandes empresas juegan un papel central. Estas son responsables en un grado importante de la emisión de gases de efecto invernadero, del vertido de líquidos tóxicos, de la generación y acumulación de residuos contaminantes, por citar sólo algunos de los más relevantes. De hecho, su presencia destacada en áreas altamente contaminantes como las industrias extractivas (hidrocarburos, minería, etc.) las coloca en una posición de primer orden entre los agentes responsables (Petras y Veltmeyer, 2007: 228). Las multinacionales petroleras son un ejemplo paradigmático en este sentido. Podríamos citar casos como el de Texaco en Ecuador, responsable del vertido de "más de 19.000 millones de galones de agua residual", la quema "diaria de dos millones de metros cúbicos de gas" que provocan efecto invernadero y lluvia ácida, el "derrame de más de 16 millones de galones de crudo" y la corresponsabilidad en el aumento de casos de cáncer y de abortos espontáneos en sus zonas de operación (Bárcena y Lago, 20008:157).

2. Destrucción biodiversidad. La destrucción del hábitat y de la biodiversidad es otro de los impactos ambientales de gran trascendencia, que implica daños a la fauna y a la flora de un territorio, producto de prácticas depredadoras que generan desforestación, desaparición de especies, inundación de tierras, etc.

El crecimiento de la conciencia ecológica y de la consiguiente presión social ha obligado a muchas de las transnacionales a redefinir su discurso y adaptarse a los nuevos tiempos haciendo uso del "marketing ecológico" que en muchos casos es efectivo para mejorar su prestigio y "aumentar sus ventas". Sin embargo, paralelamente, algunas de estas siguen ejerciendo fuertes presiones para bloquear iniciativas internacionales en favor del medio ambiente, como ha ocurrido con el Protocolo de Kioto, que han intentado deslegitimar corporaciones como Ford, General Motors, Mobil y Unión Carbide (Teitelbaum, 2010:141).

\section{4. Área social}

La actividad de las multinacionales provoca también impactos de orden social y/o socioeconómico, que no podemos soslayar y que afectan a las comunidades, a las y los trabajadores y en muchos casos también a los y las usuarias de algunos servicios que las corporaciones prestan. 
1. Laboral. Comenzaremos identificando los impactos de orden laboral que afectan a las y los trabajadores directos e indirectos de las transnacionales. En primer lugar, nos parece apropiada la división que establece Teitelbaum entre impactos relacionados con el derecho al trabajo, es decir, a la posibilidad de desempeñar una ocupación remunerada y los "derechos en el trabajo", es decir, el derecho a trabajar en condiciones dignas (Teitelbaum, 2010:87).

Respecto al primer aspecto, el derecho al trabajo, podemos citar varios impactos negativos que genera la lógica capitalista de las multinacionales a escala internacional. Uno de ellos es la destrucción de empleo en los países del capitalismo central debido al creciente fenómeno de la deslocalización que lleva a situar los centros de trabajo en los países de la periferia, buscando aumentar sus ganancias gracias a los salarios más bajos que se pueden permitir pagar, etc. La amenaza de la deslocalización, per se, también lesiona el derecho al trabajo cuando los sindicatos de los países centrales aceptan reducir plantillas y/o condiciones laborales para evitar el cierre total de la empresa. En cuanto a los países del Sur, la promesa de la creación de puestos de trabajo en más de una ocasión implica la destrucción de empleos vinculados a la economía local que no puede competir (Teitelbaum, 2010:88-92). Además, en muchos casos, más que crear empleo en los países periféricos lo que hacen es reducirlo, ya que no crean capacidad productiva sino que compran empresas ya existentes a las que aplican fuertes reducciones de plantilla en aras de la supuesta eficiencia.

En cuanto al segundo aspecto, los "derechos en el trabajo" o las condiciones laborales, podemos citar diversos elementos que suponen un impacto negativo para el y la trabajadora. El deterioro de las condiciones laborales suele producirse de manera muy evidente cuando antiguas empresas estatales pasan a manos de grandes corporaciones privadas. Reducción salarial, fin de la estabilidad laboral, supresión de los convenios colectivos, tercerización de algunas áreas, aumento de la inseguridad laboral, discriminación por género, etnia, etc., y trabajo infantil y forzoso, son algunas de las principales denuncias que se realizan contra las multinacionales. A esto hay que añadir el ataque a los derechos sindicales como la libertad de afiliación, el respeto a la acción sindical sin represalias, etc. El caso de las denuncias en Colombia contra Coca Cola por su supuesta complicidad en el asesinato de sindicalistas en este país (Teitelbaum, 2010:105), es uno de los ejemplos más extremos en este terreno. Sin embargo, la práctica más generalizada suele ser mucho más sutil e implica acoso y/o despidos a lideres sindicales y a sus afiliados, prohibición de facto de la creación de sindicatos, etc.

2. Comunidad. Las comunidades de los lugares donde las multinacionales tienen presencia se convierten en algunos casos en sectores afectados por las prácticas de estas. Los impactos son diversos y muy diferentes en función del tipo de empresa y su dinámica. Desplazamientos de población, pérdida de tierras (Ramiro, 2011:10), impactos en la economía local (agricultura, pesca...), ruptura o deterioro del tejido social, familiar, de los vínculos afectivos, son solo algunos de los impactos comunitarios que provocan los emprendimientos de las transnacionales (Petras y Veltmeyer, 2007:226-7).

3. Género. Los impactos en las relaciones de género en realidad se pueden considerar de carácter transversal, ya que se manifiestan en diversas áreas (mercado de trabajo, ámbito reproductivo, comunitario, seguridad, etc.). Los efectos múltiples que provocan las multinacionales se suelen manifestar de manera diferenciada y con mayor virulencia contra las mujeres, por su posición subordinada en el marco del sistema económico mundial y en el contexto de las relaciones patriarcales (Parella, 2003) en el que operan las empresas. Así, por ejemplo, la explotación laboral es un impacto de primer orden que afecta a las mujeres. El reclutamiento de mujeres como fuerza de trabajo asalariada en fábricas situadas en las zonas 
francas donde las condiciones laborales suelen ser sumamente precarias, certifica esta realidad. El modelo de división internacional y sexual del trabajo impuesto por las transnacionales reserva a las mujeres trabajos en sectores de gran explotación "como el textil, el procesamiento de alimentos, los 'call-centers', etc.” (González y Ramiro, 2013:12).

Por otra parte, "la mercantilización de los servicios públicos" en muchos países y su apropiación por parte de multinacionales, "ha impactado fundamentalmente en las personas sobre las que recae el trabajo de cuidados y reproducción social, es decir, las mujeres". Los cortes de electricidad y de agua, por ejemplo, en barrios pobres ha aumentado la carga de trabajo en el hogar en "detrimento de las condiciones de salud, la calidad de vida y el tiempo libre" de las mujeres (González y Ramiro, 2013:13). La construcción de grandes obras y la llegada masiva de personal de las empresas provoca, en más de una ocasión, graves desigualdades de género por el aumento de la inseguridad, de la prostitución, de la violencia contra las mujeres, etc.

4. Usuarios. Las y los usuarios de servicios prestados por multinacionales conforman otro de los grupos sociales que en algunos casos padecen la presencia de estas empresas. Los casos más paradigmáticos se observan en corporaciones que prestan servicios públicos como la distribución de electricidad, gas, agua, telefonía... La marginación de grupos de población de barrios populares o de zonas rurales alejadas suele ser una consecuencia en algunos casos de la aplicación de la lógica del lucro que guía la práctica de estas empresas, que no consideran rentable o identifican como "grupos de riesgo" a estos sectores. Esto puede implicar impactos severos en términos de cobertura (Ramiro, 2011:10-11).

Otro impacto habitual suele ser el aumento de las tarifas del servicio, a pesar de que el discurso favorable a la privatización siempre promete que la competencia en el sector traerá una reducción del precio. A la subida de las tarifas hay que añadir, en algunos casos, las denuncias por costos indebidos, por costos de reconexión, etc. La precarización de las condiciones laborales como la tercerización, etc., suelen provocar también impactos en la calidad del servicio, como sucede en el sector de la electricidad, por ejemplo, y que se traducen en un número mayor de cortes, apagones... (Ramiro, 2011:11).

\section{5. Área cultural}

Indígenas. Los impactos culturales constituyen otro foco de análisis a la hora de evaluar la práctica de las multinacionales. Uno de los campos más paradigmáticos es el relativo a los diversos impactos que provocan en los pueblos indígenas. Las agresiones a su territorio tanto a su propiedad como a los recursos de que disponen-, a su identidad cultural y a sus modos de organización política son algunas de las violaciones más denunciadas. Berraondo (2008: 93) asegura que existe un "cuadro recurrente" de violaciones a los pueblos indígenas como la expropiación de tierras, la reubicación, la inundación de sus territorios, la discriminación, la destrucción medioambiental de su hábitat, la represión, etc. Podemos destacar los conflictos entre multinacionales y algunos pueblos indígenas de América Latina como el de los pueblos originarios de tierras bajas en Bolivia frente a las petroleras, el de las comunidades indígenas de la Amazonia peruana contra las mineras, el de los mapuches frente a corporaciones eléctricas como Endesa en Chile, etc. (Ramiro y González, 2010:189200). 
Tabla 1. Áreas y dimensiones para evaluar impactos de multinacionales

\begin{tabular}{|c|c|c|}
\hline Áreas & Dimensiones & Descripción \\
\hline \multirow{5}{*}{ 1. Económica } & 1.1. Propiedad & $\begin{array}{l}\text { Grado de mercantilización de un bien o servicio } \\
\text { público (privatización...) }\end{array}$ \\
\hline & 1.2. Soberanía & Grado de extranjerización del sector \\
\hline & 1.3. Rentabilidad empresarial & $\begin{array}{l}\text { En detrimento del erario público (subsidios, ventajas } \\
\text { fiscales...) y/o de la ciudadanía (tarifas...) }\end{array}$ \\
\hline & 1.4. Eficiencia & $\begin{array}{l}\text { Eficiencia del servicio prestado: inversión en } \\
\text { infraestructura, mantenimiento... }\end{array}$ \\
\hline & 1.5. Empresas locales & Prioridad a empresas locales o externas \\
\hline \multirow{7}{*}{ 2. Política } & 2.1. Democracia & $\begin{array}{l}\text { Apoyo a regímenes autoritarios, injerencia en } \\
\text { sistema político... }\end{array}$ \\
\hline & 2.2. Lobby & Práctica del lobby \\
\hline & 2.3. Medios de comunicación & $\begin{array}{l}\text { Influencia en los medios de comunicación, control } \\
\text { de estos... }\end{array}$ \\
\hline & 2.4. Marco jurídico & Presión para cambios legislativos favorables \\
\hline & 2.5. Corrupción & $\begin{array}{l}\text { Sobornos a poderes públicos, organismos } \\
\text { reguladores }\end{array}$ \\
\hline & 2.6. Falta de transparencia & $\begin{array}{l}\text { Presencia en paraísos fiscales, publicidad engañosa, } \\
\text { etc. }\end{array}$ \\
\hline & 2.7. Criminalización y represión & $\begin{array}{l}\text { De trabajadores/as o movimientos sociales que se } \\
\text { oponen a sus prácticas }\end{array}$ \\
\hline \multirow[b]{2}{*}{ 3. Ambiental } & 3.1. Contaminación & Aire, agua, tierra, animales, humanos \\
\hline & $\begin{array}{l}\text { 3.2. Destrucción de hábitat y } \\
\text { biodiversidad }\end{array}$ & Inundación, deforestación... \\
\hline \multirow{4}{*}{ 4. Social } & 4.1. Laboral & $\begin{array}{l}\text { Derecho al trabajo, condiciones laborales, derechos } \\
\text { sindicales... }\end{array}$ \\
\hline & 4.2. Comunidad & $\begin{array}{l}\text { Impactos estructura socio-económica local } \\
\text { (desplazamientos, expolio de tierras, etc.) }\end{array}$ \\
\hline & 4.3. Género & Precariedad laboral, inseguridad... \\
\hline & 4.4. Usuarios/as & $\begin{array}{l}\text { Calidad de servicios, cobertura, tarifas, salud y } \\
\text { seguridad... }\end{array}$ \\
\hline 5. Cultural & 5.1. Pueblos indígenas & $\begin{array}{l}\text { Agresiones a su propiedad, territorio, identidad } \\
\text { cultural, etc. }\end{array}$ \\
\hline
\end{tabular}

Fuente: Elaboración propia

\section{CONCLUSIONES}

Los impactos que provocan las empresas multinacionales son, como hemos podido enumerar en este trabajo, múltiples, muy diversos, y en muchos casos con graves consecuencias para la población afectada. La imagen idílica que algunos estudios cercanos a estas empresas intentan proyectar, se aleja por tanto de manera drástica de la realidad, en la que las corporaciones transnacionales más que como agentes de desarrollo aparecen como responsables directas de importantes vulneraciones de derechos sociales y humanos. Las corporaciones son hoy día, más que nunca, los agentes estratégicos del capitalismo y en consecuencia, las principales responsables de operar en función de los intereses de las elites y en perjuicio de la gran mayoría de la ciudadanía.

En el contexto actual de la denominada oficialmente "crisis económica", donde la estrategia de saqueo de la riqueza social y pública se ha incrementado de manera exponencial, la centralidad de las multinacionales es aún mucho mayor. Los altos beneficios 
de algunas de estas grandes corporaciones contrastan con el deterioro de las condiciones de vida de una gran parte de la sociedad, haciendo más evidente su papel marcadamente funcional a la lógica concentradora de riqueza y generadora de desigualdad y pobreza que imprime el sistema capitalista.

Siendo conscientes de esta situación, consideramos fundamental que se sigan construyendo propuestas teóricas y metodológicas que sirvan para analizar de manera crítica y rigurosa la actualidad del capitalismo y para evaluar las prácticas de las corporaciones transnacionales. La actual orientación del sistema económico internacional está otorgando mayor poder económico y político a estas empresas y por ello será cada vez más necesario contar con herramientas adecuadas para poder hacer un seguimiento de sus actuaciones.

\section{BIBLIOGRAFÍA}

ACHBAR, M. y ABBOT, J. (2009). La Corporación. Documental. Canadá: ISAAN Entertainment S.L. BALANYÁ, B. (2007). “¿Cenamos esta noche, comisario? Lobby en Bruselas”. En AA.VV. Las empresas transnacionales en la globalización. Madrid: OMAL.

BARBA, M. (2008). "Haciendo las Américas: la aventura de Iberdrola en el nuevo continente", en Boletín OMAL, 29: 1-13

BÁRCENA, I. y LAGO, R. (2008). "Deuda ecológica vs Responsabilidad social corporativa: las empresas transnacionales ante la crisis socio-ecológica", en Revista de Relaciones Laborales "Lan Harremanak", 19-II: 141-167.

BASTERRA, J. (2011). BBVA e Iberdrola miran desde lo más alto del ranking mundial, accesible en http://gara.naiz.info/paperezkoa/20110717/279401/es/BBVA-e-Iberdrola-miran-desde-mas-altoranking-mundial, (consultado 4/1/2014)

BERRAONDO, M. (2008). "Aplicación del derecho o programas de RSC para pueblos indígenas", en Revista de Relaciones Laborales "Lan Harremanak", 19 (II): 89-116.

CARRIÓN, J. y VERGER, T. (2007). “Amistades peligrosas: empresas transnacionales, poder político y poder mediático". En AA.VV. Las empresas transnacionales en la globalización. Madrid: OMAL.

CASILDA BÉJAR, R. (ed.) (2008). La gran apuesta. Globalización y multinacionales españolas en América Latina. Análisis de los protagonistas. Barcelona: Granica.

GINER, A. (2008). "Las empresas transnacionales y los derechos humanos", en Revista de Relaciones Laborales "Lan Harremanak", 19 (II): 67-87.

GONZÁLEZ, E. y RAMIRO, P. (2013). "Multinacionales, impactos y desigualdades de género", en Revista Pueblos, 55: 12-13.

GUILLÉN, M. (2006). El auge de la empresa multinacional española. Madrid: Fundación Rafael del Pino.

HERNÁNDEZ ZUBIZARRETA, J. (2008). "La Responsabilidad Social Corporativa y las empresas transnacionales: de la ética de la empresa a las relaciones de poder", en Revista de Relaciones Laborales "Lan Harremanak", 19 (II): 17-49.

HERNÁNDEZ ZUBIZARRETA, J. (2009a). “LLex mercatoria o derechos humanos? Los sistemas de control de las empresas transnacionales”. En J. Hernández Zubizarreta y R. Ramiro (eds.). El negocio de la responsabilidad. Crítica de la Responsabilidad Social Corporativa de las empresas transnacionales. Barcelona: Icaria-Antrazyt.

HERNÁNDEZ ZUBIZARRETA, J. (2009b). Las empresas transnacionales frente a los derechos humanos: historia de una asimetría normativa. Bilbao: Hegoa.

PARELLA, S. (2003). "Repensando la participación de las mujeres en el desarrollo desde una perspectiva de género", en Papers. Revista de Sociología, 69: 31-57.

PETRAS, J. y VELTMEYER, H. (2007). Juicio a las multinacionales. Inversión extranjera e imperialismo. Madrid: Editorial Popular. 
RAMIRO, P. (2009). "Las multinacionales y la Responsabilidad Social Corporativa: de la ética a la rentabilidad”. En J. Hernández Zubizarreta y P. Ramiro (eds.). El negocio de la responsabilidad. Crítica de la Responsabilidad Social Corporativa de las empresas transnacionales. Barcelona: Icaria-Antrazyt. RAMIRO, P. (2011). "El segundo desembarco: los impactos de las multinacionales españolas en América Latina”. En S. Álvarez (coord.). Convivir para perdurar. Conflictos ecosociales y sabidurías ecológicas. Barcelona: Icaria-Antrazyt. (Manuscrito facilitado por el autor antes de la publicación).

RAMIRO, P. y GONZÁLEZ, E. (2010). "Multinacionales españolas en América Latina: impactos sobre los pueblos indígenas". En A. Cruz et al. Pueblos originarios en América. Guía introductoria de su situación. Pamplona: ALDEA.

SÁNCHEZ PÉREZ, J. (2008). "El poder de las empresas multinacionales". X Coloquio Internacional de Geocrítica. Diez años de cambios en el mundo, en la geografía y en las Ciencias Sociales, 19992008. Barcelona 26-30 de mayo de 2008: Universidad de Barcelona.

SANTISO, J. (2009). "Nuevas multinacionales", en El País, accesible en http://www.elpais.com/arti culo/primer/plano/nuevas/multinacionales/elpepueconeg/20090712elpneglse_3/Tes

SKLAIR, L. (2003). Sociología del sistema global. El impacto socioeconómico y político de las corporaciones transnacionales. Barcelona: Gedisa Editorial.

TEITELBAUM, A. (2010). La armadura del capitalismo. El poder de las sociedades transnacionales en el mundo contemporáneo. Barcelona: Icaria-Antrazyt.

UHARTE, L. M. (2005). "Política social y democracia: un "nuevo" paradigma”, en Revista Venezolana de Economía y Ciencias Sociales, 11(3): 93-114.

VAN DEN EYNDE, A. (2005). Globalización: la dictadura de las multinacionales, en IAR Noticias, accesible en http://www.iarnoticias.com/secciones_2005/norteamerica/0034-poder_de_multinacionales -09may05.html

ZABALO, P. (2007). "El papel de las multinacionales en la economía globalizada". En AA.VV. Las empresas transnacionales en la globalización, Madrid: OMAL.

\section{Breve currículo:}

\section{Luis Miguel Uharte Pozas}

Doctor en Ciencias Sociales (Estudios Latinoamericanos) por la Universidad Complutense de Madrid. Profesor del departamento de Antropología Social de la Universidad del País Vasco (UPV/EHU). Miembro del Grupo de Investigación 'Parte Hartuz' y del Instituto de Estudios sobre Desarrollo 'Hegoa' de la Universidad del País Vasco (UPV/EHU). Principales líneas de investigación: desarrollo e impactos de multinacionales, cambio político en América Latina, nuevos modelos de democracia, políticas sociales y políticas públicas. 